\title{
EVALUACIÓN DEL DESEMPEÑO DE LOS EXPOSITORES EN FERIAS INTERNACIONALES
}

\section{EXHIBITOR PERFORMANCE EVALUATION IN INTERNATIONAL FAIRS}

\author{
Enrique Angles Sánchez \\ Doctorando en Educación - Universidad Técnica de Berlín, Alemania; Maestría en Administración - Universidad Nacional Mayor de San \\ Marcos, Lima, Perú. - Email: eangles@yahoo.com (Autor Corresponsal)
}

[Recibido: 15/12/2016 Aceptado: 16/02/2017]

\begin{abstract}
RESUMEN
Las ferias internacionales son un instrumento de marketing internacional generalmente usadas por las empresas exportadoras. A pesar de sus ventajas, en el caso de las empresas exportadoras peruanas se evidencia una limitada participación como expositores, y las pocas que participan no llegan a evaluar el desempeño de su participación. El presente estudio se orienta a probar si las empresas expositoras evalúan su desempeño ferial principalmente mediante las ventas obtenidas, tal como lo señala la literatura existente. Para ello se trabajó un modelo de desempeño ferial a partir de las ventas. Para el análisis empírico cuantitativo, se aplicó una encuesta específica a expositores peruanos de las dos más importantes ferias de los sectores confecciones, moda y artesanía. Los resultados demostraron que las ventas, a partir de su participación ferial, tienen una correlación positiva y significativa con la evalxuación de su desempeño ferial.
\end{abstract}

\section{PALABRAS CLAVE}

Medición, desempeño, ferias internacionales, expositores.

\begin{abstract}
International fairs are an international marketing tool generally used by exporting companies. Despite its advantages, in the case of the Peruvian exporting companies a limited participation as exhibitors is evidenced, and the few that participate do not come to evaluate the performance of their participation. The present study is aimed at proving if the exhibiting companies evaluate their fair performance mainly through the sales obtained, as the existing literature indicates. For this, we worked a fair performance model from the sales. For the quantitative empirical analysis, a specific survey was applied to Peruvian exhibitors of the two most important trade fairs in the clothing, fashion and handicraft sectors. The results showed that the sales, from their participation in the fair, have a positive and significant correlation with the evaluation of their fair performance.
\end{abstract}

\section{KEYWORDS}

Measurement, performance International fairs, exhibitors.

Como Citar: López, T. (2017). Análisis tributario de las comisiones por servicios de los agentes intermediarios textiles. Quipukamayoc 25(48), 77-82. doi: http://dx.doi.org/10.15381/quipu.v25i48.13995

\section{INTRODUCCIÓN}

Cuando las empresas exportadoras deciden participar en ferias internacionales - que según Kirchgeorg, Springer y Kastner (2010) son eventos que se llevan a cabo en días específicos, donde un número grande de empresas presentan sus productos - es porque las consideran como una alternativa importante para poder buscar y conquistar mercados externos, algo imprescindible en la actual economía globalizada (Betz, 2008). Esta participación influiría en el desempeño exportador por tres razones:

1) Permite examinar el potencial exportador.

2) Genera contactos y otras iniciativas de cooperación con empresas distribuidoras en mercados del exterior.

3) permite investigar mercados por medio de evaluaciones comparativas de los ofrecimientos de otros exportadores (Terpstra y Sarathy, 1997).

Sin embargo, a pesar de estas ventajas, las exportadoras peruanas tienen una limitada participación. Sashi y Perretty (1992) mencionan que parte de la justificación se basa en que conlleva un nivel alto de inversión (previa y durante la realización), lo que genera escepticismo, especialmente si no se conoce como medir los resultados conseguidos con su participa-

(c) Los autores. Este artículo es publicado por la Revista Quipukamayoc, Universidad Nacional Mayor de San Marcos. Este es un artículo de acceso abierto, distribuido bajo los términos de la Licencia Creative Commons Atribución-NoComercial-Compartirlgual 4.0 Internacional.(http://creativecommons.org/licenses/by-nc-sa/4.0/), que permite el uso no comercial, distribución y reproducción en cualquier medio, siempre que la obra original sea debidamente citadas. 
ción para justificar dicha inversión y evaluar la eficacia de la participación (Herbig, O’Hara y Palumbo 1994; Tafesse y Korneliussen, 2011).

A pesar de ello y casi de manera intuitiva, las empresas exportadoras peruanas que participan regularmente en ferias internacionales las consideran como parte de sus estrategias de marketing. Por lo tanto, la evaluación del desempeño de un expositor en ferias es imprescindible, si lo que se desea es justificar la inversión realizada. En tal sentido, nuestra investigación plantea como hipótesis principal la existencia de una relación positiva entre las ventas y el desempeño ferial de las empresas expositoras peruanas durante su participación en ferias internacionales.

Esta hipótesis se sostiene de los hallazgos de Hansen (2004) y Kim (2005), que señalan que dentro de las principales dimensiones orientadas a la evaluación del desempeño ferial, aquellas relacionadas con la venta son quizás las más críticas. Empíricamente la evidencia demostró que las empresas expositoras peruanas si determinan su desempeño en las ferias internacionales a través de las ventas obtenidas. Como todo estudio empírico, las limitaciones se centraron básicamente en la obtención de una muestra estadísticamente significativa, dado que el universo de empresas expositoras peruanas es limitado. A pesar de ello, se cumplieron los criterios requeridos para la estimación del modelo estructural multivariado (SEM).

Una feria internacional reúne, en un mismo lugar y en cortos periodos de tiempo, a empresas exportadoras de distintos países y compradores internacionales, facilitando una rápida comparación entre los productos de diversa procedencia, a un costo accesible, generando adicionalmente oportunidad de ventas (Dekimpe, François, Goplakrishna, Lilien, Van Den y Bulte, 1997; O'Hara, Palumbo y Herbig, 1993 y Blythe, 2010).

Sin embargo, la evaluación del desempeño ferial no ha podido ser incorporada en las prácticas de gestión de las empresas debido a que muchas empresas no fijan objetivos de participación; o si los fijan, éstos suelen ser muy heterogéneos lo cual dificulta su evaluación; o porque la participación en las ferias se complementa con otras herramientas de marketing, lo cual complica el cálculo de su rentabilidad real dadas las interacciones resultantes (Mesonero y Garmendia 2004).

Para muchas empresas, todo lo anterior se reduce a un único objetivo que determina el desempeño como expositor: las ventas, (Smith, Gopalakrishna y Smith, 2004). Bonoma (1983) ya había clasificado los objetivos en una feria como "objetivos de ventas" y "objetivos de no-ventas" (por ejemplo, chequear el mercado para la aceptación de un producto, localizando potenciales distribuidores, fortalecer las relaciones con los clientes actuales y potenciales). Sobre esa base, Kerin y Cron (1987), llegaron a clasificar a las empresas expositoras por su alto o bajo desempeño, basados en objetivos de venta y de no-venta.

En un primer intento para desarrollar una evaluación más estructurada, Volmer (2003) se orientó a analizar los procedimientos que utilizan las empresas para la medición del éxito de sus participaciones en las ferias.

Sin embargo, fue finalmente Hansen (2004) el primero que planteó un modelo que incluye una dimensión basada en los resultados de ventas, además de otras cuatro dimensiones basadas en el comportamiento.

Los ajustes al modelo de Hansen fueron realizandos principalmente por Tafesse (2009). evaluando el impacto de algunas variables en el desempeño de los expositores y Keswell (2010) propuso un modelo conceptual que permite vincular las actividades de marketing ferial y relacionarlas con los resultados de marketing y de ventas, medidos como indicadores financieros.

La literatura refuerza nuestro planteamiento de la existencia de una relación positiva y significativa entre las ventas y la evaluación del desempeño ferial. Destacamos 5 argumentos:

1. Conseguir ventas. Este es uno de los objetivos más importantes de una feria. Muchos pedidos se cierran en el propio stand. En concreto, se considera que, para cerrar una venta, la actividad ferial es muy eficiente, con una influencia solo por detrás de la recomendación de conocidos y la venta personal (Shipley, Egan y Wong, 1993).

2. Realizar nuevos contactos con potenciales compradores. Hacer contactos con nuevos clientes es otro de los objetivos más valorados por los expositores (Mesonero y Garmendia, 2004; Shipley et al., 1993). En una feria se persigue contactar con un público objetivo de calidad y con una buena predisposición hacia los productos presentados (Cavanaugh, 1976). Muchos visitantes no son sólo compradores sino personas influyentes en la decisión de sus respectivas empresas, y estos son más difíciles de conocer y contactar (Dekimpe et al., 1997).

3. Comunicación de las ventajas del producto. Incluye la presentación 
y demostración del producto a los clientes potenciales en el propio stand. Este contacto cara a cara con los clientes favorece el proceso de venta (Jiménez, Cazorla y Linares, 2002).

4. Introducir un nuevo producto. La feria permite dar a conocer un nuevo producto en un espacio único a multitud de clientes potenciales de calidad, (Jiménez et al., 2002). El lanzamiento sería mucho más costoso si tuviese que realizarse de forma independiente a la feria, ya que la inversión publicitaria necesaria para dar a conocer un nuevo producto es mucho mayor (Navarro, 2001).

5. Introducirse en nuevos mercados. Cuando se participa en misiones y ferias en el extranjero o en nuevas zonas geográficas dentro de un país, las empresas encuentran en estos eventos uno de los principales medios de aproximación a los nuevos mercados.

Las ferias juegan un papel especialmente importante en los mercados internacionales, ya que reúnen a empresas vendedoras y compradoras en un mismo punto.

\section{MATERIAL Y MÉTODOS}

Para llevar a cabo el contraste de la hipótesis planteada, la aplicación empírica se centró en la edición 2010 de las ferias internacionales "Perú Moda" (sector textil y confecciones) y "Perú Gift" (artesanías). Los datos relevantes para el estudio se obtuvieron a partir de cuestionarios dirigidos, trabajando con la población total de empresas (44) que habían venido participando de manera continua en ambas ferias, como una manera de orientarnos específicamente a los expositores recurrentes. Todos los ítems fueron medidos en una escala de 5 puntos Likert.
La variable independiente Ventas, se mide a través de los indicadores nivel de ventas a los actuales clientes, nivel de ventas a los nuevos clientes, y resultado de la introducción de productos nuevos durante la feria. La variable dependiente Desempeño Ferial, se mide a través de los indicadores valorización del desempeño ferial de la empresa expositora, satisfacción del expositor con su desempeño, y posibilidad de inscripción en la siguiente edición de la feria. Cada uno de estos indicadores ha sido incorporado en una pregunta del cuestionario aplicado. Se incorporó además como variable de control al tamaño de la empresa. En consecuencia, el instrumento de recolección contiene seis ítems para evaluar las percepciones de los expositores de su desempeño ferial, lo que ha sido validado ya en estudios realizados en ferias como SIAL (París) y ANUGA (Colonia), ambas orientadas a alimentos.

Hemos utilizado un modelo de ecuaciones estructurales para contrastar la

Tabla 1.

Resultados Generales de la Encuesta Aplicada Fuente: Elaborado por Autor

\begin{tabular}{|c|c|c|c|c|c|c|}
\hline & \multicolumn{3}{|c|}{ Ventas } & \multicolumn{3}{|c|}{ Desempeño ferial } \\
\hline & $\begin{array}{l}\text { P1. } \\
\text { ¿Cómo } \\
\text { conside- } \\
\text { ra que } \\
\text { fueron } \\
\text { sus ventas } \\
\text { en esa } \\
\text { feria para } \\
\text { quienes } \\
\text { son sus } \\
\text { clientes } \\
\text { actuales? }\end{array}$ & $\begin{array}{l}\text { P2. ¿Le } \\
\text { sirvió la } \\
\text { partici- } \\
\text { pación en } \\
\text { esa feria } \\
\text { para tener } \\
\text { nuevos } \\
\text { clientes? }\end{array}$ & $\begin{array}{l}\text { P3. ¿Fue } \\
\text { útil para } \\
\text { introdu- } \\
\text { cir y/o } \\
\text { evaluar } \\
\text { reaccio- } \\
\text { nes a los } \\
\text { productos } \\
\text { nuevos } \\
\text { suyos que } \\
\text { presentó? }\end{array}$ & $\begin{array}{l}\text { P4. Si } \\
\text { usted } \\
\text { tuviera } \\
\text { que dar } \\
\text { una valo- } \\
\text { rización } \\
\text { sobre el } \\
\text { desempe- } \\
\text { ño de su } \\
\text { empresa } \\
\text { exposito- } \\
\text { ra en la } \\
\text { feria, ésta } \\
\text { sería: }\end{array}$ & $\begin{array}{l}\text { P5. } \\
\text { Hablando } \\
\text { en forma } \\
\text { general, } \\
\text { ¿qué tan } \\
\text { satisfecho } \\
\text { está usted } \\
\text { con el } \\
\text { desempe- } \\
\text { ño gene- } \\
\text { ral de su } \\
\text { empresa } \\
\text { logrado } \\
\text { en la } \\
\text { feria? }\end{array}$ & $\begin{array}{l}\text { P6. ¿Tiene } \\
\text { usted in- } \\
\text { tenciones } \\
\text { de volver } \\
\text { a presen- } \\
\text { tarse en la } \\
\text { próxima } \\
\text { edición de } \\
\text { la feria? }\end{array}$ \\
\hline Media & 3,41 & 3,55 & 3,89 & 3,77 & 3,93 & 4,39 \\
\hline $\begin{array}{l}\text { Error } \\
\text { estándar }\end{array}$ & 0,104 & 0,110 & 0,081 & 0,085 & 0,105 & 0,109 \\
\hline
\end{tabular}

hipótesis planteada, lo que se ajusta a nuestro objetivo de probar conceptos (Hair, Black, Babin y Anderson, 2010). En la evaluación del modelo de medida, se ha comprobado la fiabilidad para evaluar su consistencia interna a través del Alfa de Cronbach. Se utilizaron los programas MS Excel 13 y SPSS 22 para el tratamiento y análisis del modelo.

\section{RESULTADOS}

En la Tabla 1, podemos apreciar que a nivel de la variable Ventas, los indicadores se situaron a niveles entre 3,41 a 3,89 (en la escala 1-5). A nivel de la variable Desempeño ferial, los indicadores se situaron a niveles entre 3,77 a 4,39. Los errores estándar no fueron excesivos.

Sin embargo, en la medida que estos valores individuales pueden ser útiles solo para fines descriptivos, se desarrollaron correlaciones bivariadas que nos proporcionaron una primera aproximación a la estimación de la 
Tabla 2 .

Correlaciones bivariadas (Pearson)

Fuente: Elaborado por Autor

* La correlación es significativa en el nivel 0,05 (2 colas).

**. La correlación es significativa en el nivel 0,01 (2 colas).

\begin{tabular}{|c|c|c|c|c|c|c|c|}
\hline & & P.1 & P. 2 & P.3 & P.4 & P.5 & P.6 \\
\hline \multirow[t]{2}{*}{ P.1 } & Pearson & 1 & & & & & \\
\hline & Sig. (bil.) & & & & & & \\
\hline \multirow[t]{2}{*}{ P.2 } & Pearson & 0,238 & 1 & & & & \\
\hline & Sig. (bil.) & 0,119 & & & & & \\
\hline \multirow[t]{2}{*}{ P.3 } & Pearson & 0,065 & $-0,135$ & 1 & & & \\
\hline & Sig. (bil.) & 0,674 & 0,383 & & & & \\
\hline \multirow[t]{2}{*}{ P.4 } & Pearson & 0,184 & $0,364^{*}$ & $-0,317^{\star}$ & 1 & & \\
\hline & Sig. (bil.) & 0,233 & 0,015 & 0,036 & & & \\
\hline \multirow[t]{2}{*}{ P.5 } & Pearson & $0,301^{*}$ & 0,121 & 0,041 & $0,552^{\star *}$ & 1 & \\
\hline & Sig. (bil.) & 0,047 & 0,435 & 0,792 & 0,000 & & \\
\hline \multirow[t]{2}{*}{ P.6 } & Pearson & 0,142 & $-0,056$ & $-0,064$ & 0,220 & 0,146 & 1 \\
\hline & Sig. (bil.) & 0,359 & 0,717 & 0,680 & 0,151 & 0,343 & \\
\hline
\end{tabular}

explicatividad de la variable dependiente a través de la variable independiente. os resultados mostraron que, para el caso de los indicadores de la variable ventas, existían correlaciones negativas entre ellas, lo que implicaba evaluar la fiabilidad de la variable (ver Tabla 2).

De igual forma, el indicador proveniente de la pregunta 6 era poco significativo respecto a la variable Ventas y sus componentes.

En cuanto a la fiabilidad de la variable Ventas, el Alfa de Cronbach es

de 0,186 . Se procedió a eliminar del modelo al indicador 3 de la Variable Ventas, incrementando el Alfa de Cronbach a 0,384. Igualmente, respecto a la variable de Desempeño ferial, el Alfa de Cronbach es 0,547. Este valor pudo subir hasta $0,701 \mathrm{ex}-$ trayendo del modelo al indicador 6, de la variable Desempeño ferial.

Adicionalmente al procedimiento anterior, se desarrolló el análisis factorial confirmatorio. En el caso de las ventas, los indicadores 1 y 2 se agruparon en un solo componente (utilizando la matriz de transformación de compo-

\section{Tabla 3.}

Resultados del modelo estructural Fuente: Elaborado por Autor

\begin{tabular}{|c|c|c|c|c|}
\hline & \multirow{2}{*}{ Modelo } & \multicolumn{2}{|c|}{ Coeficientes no estandarizados } & \multirow{2}{*}{ Sig. } \\
\hline & & B & Error estándar & \\
\hline \multirow{3}{*}{1} & (Constante) & 3,313 & 1,015 & 0,002 \\
\hline & Ventas1 & 0,256 & 0,169 & 0,137 \\
\hline & Ventas 2 & $-0,144$ & 0,168 & 0,397 \\
\hline
\end{tabular}

nente), mientras que el indicador 3 , se mantiene de forma individual al pertenecer a otro concepto.

Habiendo, por tanto, replanteado y ajustado el modelo estructural, su representación gráfica puede verse en la Figura 1.

Las nuevas variables presentaron situaciones de normalidad, en el caso de Ventas 1, Ventas 2. Se evidenció claramente que la única variable que independientemente mostró correlación con el Desempeño, fue la variable Ventas 1 (0,344 al 0,05 de significancia). El modelo replanteado presentó un R2 de 0,132 y un R2 ajustado de 0,09. Se realizó el Análisis de la Varianza que arrojó como resultado un valor $\mathrm{F}$ de 3,129.

En tal sentido, se puede señalar de manera contundente que la única variable con cierto nivel de significancia es Ventas1 (es decir, la percepción acerca de las ventas para los clientes actuales, y la posibilidad de contar con nuevos clientes).

Ventas2 (es decir, la utilidad para introducir y/o evaluar reacciones a los productos nuevos presentados) demostró no ser explicativa del desempeño ferial. Los resultados generales pueden apreciarse en la Tabla 3.

Conforme a los resultados hallados, podemos señalar que el nivel de ventas a clientes actuales y el nivel de ventas a nuevos clientes influyeron en la valorización de la empresa expositora de su participación y también influyeron en la satisfacción del expositor con su desempeño en la feria. Este resultado está alineado a lo señalado por Kim (2005), acerca de que "conseguir ventas" es uno de los objetivos más importantes de una feria. El autor presentó algunos resultados donde se reportaba que realizar ventas era una 
de las principales categorías de lo que las empresas expositoras deseaban ganar en una feria internacional. La categoría "realizar ventas" incluía conseguir nuevos negocios (Ventas 2 para esta investigación) y hacer ventas (Ventas 1 en nuestro caso).

La no contundencia de los resultados del presente estudio en el Perú (nivel de significancia no llega al $1 \%$ ), ha sido evidenciada previamente en otros estudios, aunque lo real es que no hay literatura previa que demuestre fehacientemente la eficacia de una feria como herramienta de actividad de marketing para generar ventas (Keswell, 2010).

Del mismo modo, Blythe (2010) consideraba que la no fiabilidad de los resultados en estos estudios se debe en gran parte al hecho de que pocos visitantes a las ferias tienen un papel relevante en el proceso de compra, e incluso los que lo hacen no se encuentran participando en el ciclo de compra, por lo cual no están en la capacidad de hacer cualquier compromiso in situ.

Los expositores feriales con frecuencia actúan como si no fueran conscientes de que la mayoría los visitantes no son los compradores. Como resultado, algunos expositores dejan la feria sin haber logrado sus objetivos, $\mathrm{y}$ muchos visitantes se vienen quejando sobre el "enfoque de los expositores en demasía hacia las ventas". Por otro lado, la introducción de productos nuevos durante la feria no influyó en la valorización de la empresa expositora de su participación y tampoco influyó en la satisfacción del expositor con su desempeño en la feria, lo que corrobora lo señalado por Tanner y Chonko (1995), resaltaron que el objetivo principal de los expositores de ferias eran las ventas (alrededor

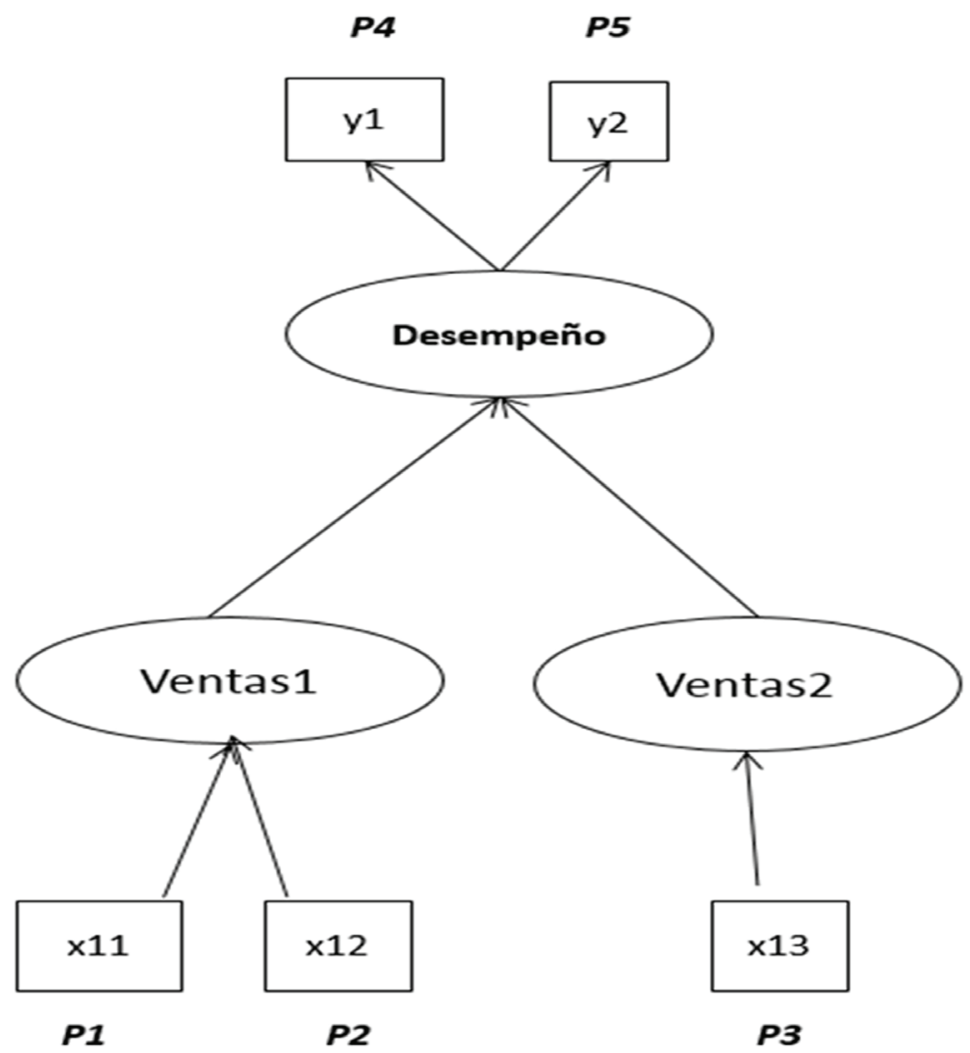

Figura 1. Modelo Estructural Fuente: Elaborado por Autor

del 62\%), de la introducción de nuevos productos o generación de nuevos negocios.

\section{DISCUSIÓN}

Las empresas expositoras peruanas evalúan su desempeño ferial principalmente a través del nivel de ventas a clientes actuales y a nuevos clientes, los cuales influyen en la valorización de la empresa expositora sobre su participación y también en la satisfacción de la misma con su desempeño en la feria.

La introducción de productos nuevos durante la feria no influye en la valorización de la empresa expositora de su participación y ni tampoco influye en la satisfacción de la misma con su desempeño en la feria, lo que está de acuerdo con algunos estudios previos que resaltaron que el objetivo principal de los expositores de ferias era las ventas, dejando de lado la introducción de nuevos productos o generación de nuevos negocios.

Las empresas expositoras peruanas tienen una orientación de marketing transaccional antes que de marketing de relaciones, considerando a las ferias como un evento a corto plazo, pues las ventas y el desempeño ferial se relacionan positivamente. Investigaciones anteriores habían determinado que las ferias son eventos para la venta a corto plazo, lo cual se cumple para el caso de las empresas 
expositoras peruanas. El marketing de relaciones implica que los gerentes de las empresas expositoras realicen seguimiento intenso a los potenciales clientes que visitaron su stand, y que son prospectos de venta. La mayoría de potenciales ventas se concretan una vez finalizada la feria, dado que el potencial comprador debe evaluar el producto ofrecido si cumple sus requerimientos, compartir la decisión de compra con sus directivos y decidir una compra.

\section{REFERENCIAS BIBLIOGRÁFICAS}

Betz, M. (2008). Messen als Instrument zur Geschäftsanbahnung auf Industriegütermärkten. Universidad de San Galo, Schesslitz, Suiza: ROSCH-Buch

Blythe, J. (2010). Trade fairs as communication: a new model. Journal of Business \& Industrial Marketing, 25(1), 57-62.

Bonoma, T. V. (1983). Get More Out Of Your Trade Show. Harvard Business Review, 61(1), 75-83.

Cavanaugh, S. (1976). Setting Objectives and Evaluating the Effectiveness of trade Show Exhibits. Journal of Marketing, 40 (4), p. 100-103.

Dekimpe, M. G., François, P., Goplakrishna, S., Lilien, G. \& Van Den Bulte, C. (1997). Generalizing About Trade Show Effectiveness: A Cross-National Comparison. Journal of Marketing, 61(4), 55-64.

Hair, J., Black, W., Babin, B. \& Anderson, R. (2010). Multivariate data analysis. (7ma ed.). Englewood Cliffs: PEARSONS
Hansen, K. (2004). Measuring Performance at Trade Shows. Scale Development and Validation. Journal of Business Research, 57(1), 1-13.

Herbig, P., O'Hara, B. \& Palumbo, F. (1994). Measuring Trade Show Effectiveness: An Effective Exercise? Industrial Marketing Management, 23(2), 165-170.

Jiménez, J. F., Cazorla, I. M. \& Linares, E. (2002). Ferias Comerciales en España. Un análisis sectorial. Distribución y Consumo, 12(61), 61-71.

Keswell, D. (2010). The effectiveness of trade show marketing capabilities on the financial performance of South African companies. Universidad de Pretoria, Gordon Institute of Business Science.

Kerin, R.A. \& Cron, W.L. (1987). Assessing trade show functions and performance: an exploratory study. Journal of Marketing, 51(3), 87-94.

Kim, Y. K. (2005). Korean Exhibitors' Behaviour in Overseas Exhibitions. Journal of Hospitality, Tourism, \& Leisure Science, 10(1), 1-18.

Kirchgeorg, M., Springer, C. \& Kast-ner, E. (2010). Objectives for successfully participating in trade shows. Journal of Business \& Industrial Marketing, 25(1), 63-72.

Mesonero de Miguel, M. \& Garmendia, F. (2004). Comunicaciones integradas feriales o cómo planificar con éxito una feria industrial. Revista de Dirección y Administración de Empresas, 10 (11), p. 109-129.
Navarro, F. (2001). Estrategias de marketing ferial. Madrid: Editorial ESIC.

O’Hara, B., Palumbo, F. \& Herbig, P. (1993). Industrial Trade Shows Abroad. Industrial Marketing Management, 10 (11), 109-129.

Sashi, C. M. \& Perretty, J. (1992). Do Trade Shows Provide Value? Industrial Marketing Management, 21(3), 249-255.

Shipley, D., Egan, C. \& Wong, K. S. (1993). Exhibiting Strategy and Implementation. International Journal of Advertising, 12(2), 117-130.

Smith, T. M., Gopalakrishna, S. \& Smith, P. M. (2004). The complementary effect of trade show attendance on personal selling. International Journal of Research in Marketing, 21 (1), p. 61-76.

Tafesse, W. \& Korneliussen, T. (2011). The Dimensionality of Trade Show Performance in an Emerging Market. International Journal of Emerging Markets, 6 (1), 38-49.

Tanner, J. \& Chonko, L. (1995). Trade Show Objectives, Management, and Staffing Practices. Industrial Marketing Management, 24(4), 257-264.

Terpstra, V. \& Sarathy, R. (1997). International Marketing. (7ma ed.). Fort Worth: Dryden Press.

Volmer, K. (2003). Beurteilung von Messebeteiligungen auf Basis der Effi-zienz am Beispiel des ITB-Messeaufritts 2003 der Norwegian Cruise Line. (Tesis de maestría). Harz University, Wernigerode, Alemania. 